\title{
Modelling the Rheological Properties of Bituminous Binders Using Mathematical Equations.
}

\begin{abstract}
This study investigates the advantages and drawbacks of several mathematical models that fit or describe linear viscoelastic rheological data of unaged and aged unmodified bitumens and polymer-modified bitumens. The complex modulus data is collected by means of a dynamic shear rheometer (DSR) within the LVE response. The result indicates that the models can be used to satisfactorily fit the rheological properties of unmodified bitumens. In general, the Generalised Modified Sigmoidal Model is the most outstanding model, followed by the Sigmoidal Model, Christensen, Anderson and Marasteanu (CAM) Model and Christensen Model and Anderson (CA) Model. However, all models suffer from similar drawbacks in that they are unable to describe the rheological properties of unaged polymer-modified bitumens precisely due to the presence of semi-crystalline and elastomeric structures in the binders and render a breakdown in the time temperature superposition principle (TTSP).
\end{abstract}

Keyword: Modelling; Linear viscoelastic region; Bitumen; Polymer-modified bitumen; Goodness-of-fit 